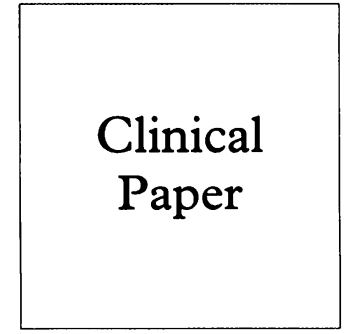

\title{
Syphilis seroprevalence among pregnant women and its role as a risk factor for stillbirth in Maputo, Mozambique
}

\author{
Elena Folgosa, Nafissa Bique Osman, Carlos Gonzalez†, Inga Hägerstrand, \\ Staffan Bergström, Åsa Ljungh
}

\begin{abstract}
Objective: To elucidate the role of current syphilis as a risk factor for foetal death.
Methods: Sera were obtained from 57 women with third trimester foetal death (cases) and 58 women with foetus alive (controls) matched for age and parity. All sera reactive in qualitative Rapid Plasma Reagin (RPR) analyses were tested with serial twofold dilutions to determine endpoint flocculation titres and tested with the micro-haemagglutination assay for Treponema pallidum (MHA-TP). Placental biopsies were sectioned and stained by haematoxylin-eosin and Warthin-Starry for light microscopy.

Setting: Central Hospital, in Maputo, Mozambique, from January 1990 to June 1991.

Results: The MHA-TP was reactive in $42 \%$ of cases and in $12 \%$ of controls (OR $=5 \cdot 3 ; 95 \% \mathrm{CI}$ : $1 \cdot 9-15 \cdot 4)$. The RPR card test was reactive at the dilution of $1: 32$ or greater in $28 \%$ of cases and in $7 \%$ of controls. All these results were confirmed by MHA-TP (OR $=5 \cdot 3 ; 95 \%$ CI: $1 \cdot 5-15 \cdot 4)$. In $9 / 28$ (32\%) MHA-TP seroreactive women ( 7 cases and 2 controls) placental morphological changes indicated syphilitic infection.

Conclusion: MHA-TP seroreactivity and high titre RPR were associated with stillbirth. Morphological changes presumptive of syphilis infection were found in $32 \%$ placentas histologically studied. Syphilis is a risk factor for foetal death in Maputo, Mozambique.

(Genitourin Med 1996;72:339-342)
\end{abstract}

Keywords: syphilis; seroprevalence; pregnancy; Maputo

Department of

Microbiology, Faculty

of Medicine, Eduardo

Mondlane University,

Maputo, Mozambique

E Folgosa

Department of

Obstetrics and

Gynecology, Maputo

Central Hospital,

Maputo, Mozambique

N Bique Osman

Department of

Pathology, Faculty of

Medicine, Eduardo

Mondlane University,

Maputo, Mozambique

C Gonzalez

Department of

Pathology, University

Hospital, S-223 62

Lund, Sweden

I Hägerstrand

Division of

International Health

Care (IHCAR),

Karolinska Institute,

5-17177 Stockholm,

Sweden

$S$ Bergström

Department of

Medical Microbiology,

Lund University, S-223

62 Lund, Sweden

Å Ljungh

†Deceased August 1994.

Address correspondence to: Prof $\AA$ L Ljungh, Departmen of Medical Microbiology, Sölvegatan 23, S-223 62, Lund, Sweden.

Accepted for publication 9 July 1996

\section{Introduction}

Reproductive tract infections are well known as a threat to the outcome of pregnancy. Syphilis during pregnancy may cause abortion, stillbirth, preterm birth or congenital disease in at least $5 \%$ of cases in which pregnancy survives beyond twelve weeks. ${ }^{1}$ A case control study of stillbirths in 1979 in Lusaka, Zambia showed that sera from $39 \%$ of 262 women with stillborn infants were reactive with the non-treponemal test Rapid Plasma Reagin (RPR) as compared with $15 \%$ of 261 control women $(\mathrm{p}<0.01) .^{2}$ In Maputo, Mozambique in 1983, Axemo et al ${ }^{3}$ reported syphilis in $8 \%$ of 168 women with late foetal death.

Recently, Lindstrand et al found an association between syphilis seroreactivity and midtrimester pregnancy miscarriage. ${ }^{4}$ An earlier study among pregnant women in Mozambique by Liljestrand $e t$ al ${ }^{5}$ found $6.3 \%$ syphilis seroprevalence (ranging from $1.6 \%$ to $9.3 \%$ ) in pregnant women. In 1992, studies of pregnant women in rural areas of two provinces of Mozambique revealed serological evidence of present or recently past syphilis ranging from $12 \%$ to $14 \cdot 6 \%{ }^{67}$

The aim of the present study was to elucidate the role of current syphilis as a risk factor for foetal death in Maputo, Mozambique.

\section{Materials and methods}

From January 1990 to June 1991, a casecontrol study was undertaken in Maputo. One group of 58 women with clinically diagnosed third trimester foetal death (cases), was recruited at Maputo Central Hospital to which they had been referred from peripheral antenatal care (ANC) clinics. A second group (controls) comprised 58 women matched for age (within five years) and parity $(0,1-3, \geqslant 4)$ with cases, was recruited among women in labour with foetus alive at Maputo Central Hospital.

Fifteen $\mathrm{ml}$ of blood were collected from each woman and $2 \mathrm{ml}$ of blood from the umbilical cord of the infants (stillborns and liveborns). Two serological assays were used: one non-treponemal test (Rapid Plasma Reagin-RPR Slide Test, BIOMERIEUX, Marcy L'Etoile, France) and one Treponemal Test (Microhaemagglutination Assay for Antibodies to Treponema pallidum, MHA-TP, AMES, Elkart, USA). All sera were qualitatively analysed (undiluted) with RPR following the manufacturer's instructions. Undiluted sera showing flocculation in RPR were further tested in serial twofold dilutions in saline $(1: 2$ up to $1: 1028)$ to determine serum endpoint titres. Results were reported as the highest dilution giving flocculation.

All RPR-reactive sera (diluted or undiluted) were qualitatively analysed by MHA-TP, to exclude false positive non-treponemal reactivity. The procedures for MHA-TP analysis followed the manufacturer's instructions.

Biopsy specimens of the placental disk were fixed in $4 \%$ formaldehyde, paraffin-embed- 
Table 1 Qualitative treponemal (MHA-TP) and non-treponemal (RPR) tests for women with late foetal death and controls, and for stillborns and liveborns

\begin{tabular}{lllll}
\hline & Cases & Controls & $O R$ & $C I$ \\
\hline Women sera & $\mathrm{N}=57(\%)$ & $\mathrm{N}=58(\%)$ & & \\
RPR REAC & $29(51)$ & $12(21)$ & $4 \cdot 0$ & $1 \cdot 6-9 \cdot 8$ \\
MHA-TP REAC & $24(42)$ & $7(12)$ & $5 \cdot 3$ & $1 \cdot 9-15 \cdot 4$ \\
Infants sera & $\mathrm{N}=21(\%)$ & $\mathrm{N}=53(\%)$ & & \\
RPR REAC & $8(38)$ & $11(21)$ & $2 \cdot 4$ & $0 \cdot 7-8 \cdot 1$ \\
MHA-TP-REAC & $7(33)$ & $6(11)$ & 3.9 & $1 \cdot 0-16 \cdot 2$ \\
\hline
\end{tabular}

$\mathrm{N}=$ Number of sera tested. $\mathrm{REAC}=$ reactive.

ded, sectioned and stained by haematoxylineosin and by Warthin-Starry, for light microscopy. Twenty-eight placentas were examined for chronic villitis, acute villitis, villous immaturity, proliferative vascular changes, chorioamnionitis and presence or absence of plasma cells in the villi and decidua. Placental weights and birthweights were recorded.

The software Epi Info ${ }^{8}$ version $5 \cdot 01 \mathrm{~b}$ was used for data entry and for the statistical analysis. Odds Ratios (OR) and 95\% confidence intervals $(\mathrm{CI})$ were calculated for comparisons of categorical variables.

\section{Results}

One hundred and fifteen sera were tested, 57 $(50 \%)$ from women with foetal death and 58 (50\%) from control women. In RPR alone, $51 \%$ and $21 \%$ of the women among cases and controls, respectively $(\mathrm{OR}=4 \cdot 0)$ were seroactive (table 1).

Seroreactivity in both RPR and MHA-TP indicating present or recently past syphilis, occurred in $42 \%$ and $12 \%$ of cases and controls, respectively. This difference is statistically significant ( $O R=5 \cdot 3$ ) (table 1$)$. Among all sera analysed, a syphilis seroprevalence of $27 \%$ was found.

RPR reverse titres equal to or above 32 and MHA-TP seroreactivity were found in 16 $(28 \%)$ of cases and in $7 \%$ of the controls (OR $=5 \cdot 3 ; 95 \% \mathrm{CI}: 1 \cdot 5-23)$. Ten of the $29 \mathrm{RPR}$ and MHA-TP reactive sera from cases (35\%) and none of the sera from control women, showed flocculation at dilution of $1: 128$ or above (table 2).

MHA-TP was non-reactive in 10/41 (24\%) RPR reactive sera. Of these sera, $5 / 29$ (17\%) and $5 / 12(42 \%)$ were from cases and from controls, respectively. For dilutions of sera above 1:8 showing flocculation in the RPR assay, $2 / 29(7 \%)$ RPR reactive sera from women with foetal death were non-reactive in MHA-TP test.

Among all the sera analysed, the calculated RPR positive predictive value was $31 / 41$
$(76 \%)$. It was higher $(83 \%)$ in cases than in controls $(58 \%)$. Among infants, 74 sera were tested, $21(28 \%)$ from stillborns and 53/74 $(72 \%)$ from liveborns. A majority of stillborns had coagulated cord blood. Nineteen of the 74 (26\%) sera from infants were RPR reactive and $18 \%$ of those, were MHA-TP reactive (table 1). Eight (38\%) of the 21 sera from the stillborns were reactive to $R P R$ as compared with $21 \%$ of those from liveborns (OR $=2 \cdot 4$ ). MHA-TP seroreactivity occurred in $33 \%$ and $11 \%$ respectively $(O R=3.9)$ (table 1$)$. RPR reactivity reverse titres equal to or above 8 were found, in $7 / 8(88 \%)$ sera from stillborns and in $3(27 \%)$ of the 11 liveborn reactive sera $(\mathrm{OR}=18 \cdot 7$; CI: 1·2-943.0).

Eighteen (75\%) placentas from cases and 7 (100\%) controls MHA-TP seroreactive were weighed. According to the standards reported by Malan et $a l^{9}$ the placental weight were above the 50th percentile in $6(33 \%)$ and 3 $(43 \%)(\mathrm{OR}=0 \cdot 7 ; 95 \% \mathrm{CI}: 0 \cdot 1-5 \cdot 6)$ cases and controls respectively; in $2(11 \%)$ cases the placental weighed was above the 90th percentile compared with none of the controls $(p=0.9)$.

Twenty-eight placentas from MHA-TP seroreactive women were histopathologically studied, 21 from cases and 7 from controls. Acute chorioamnionitis was diagnosed in 21 $(100 \%)$ of these cases and in $4(57 \%)$ controls $\left(\mathrm{X}_{2} 6.1 ; \mathrm{p}=0.01\right)$. Proliferative vascular changes (fig 1), villous immaturity (fig 2), plasma cells in the basal plate (fig 3) were visualised in $9(32 \%)$ of the 28 placentas ( 7 cases and 2 controls) (fig 1). Silver staining (Warthin-Starry) did not show spirochaetes in any of these placentas.

\section{Discussion}

In this study we found a high syphilis seroprevalence $(42 \%)$ among pregnant women with late foetal death in Maputo. This figure was three times higher than the recently reported prevalences of $15 \%$ and $12 \%$, by Vuylsteke et al, and by Cossa and co-workers in studies on pregnant women in other areas of Mozambique. ${ }^{67}$ Among control women, the syphilis seroprevalence was $6 \%$, which is within the range (2\% to $9 \%)$ found in Mozambique by Liljestrand et al in $1986 . .^{5}$ In our study, RPR and MHA-TP seroreactivities were significantly more prevalent among cases than among controls. In a previous study of causes of stillbirth in Maputo, Axemo et al found syphilis in the third place as a probable cause of foetal death, accounting for $8 \%$ of the cases. $^{3}$

Table 2 Distribution of the RPR and MHA-TP reactive sera according to RPR reactivity titres among women (cases and controls)

\begin{tabular}{|c|c|c|c|c|c|c|c|c|c|c|}
\hline & \multicolumn{10}{|c|}{$R P R$ reciprocal titres } \\
\hline & $\leqslant 2$ & 4 & 8 & 16 & 32 & 64 & 128 & 256 & 512 & 1024 \\
\hline \multicolumn{11}{|l|}{ Cases } \\
\hline RPR & & 3 & 4 & 5 & 4 & 3 & 5 & 1 & 2 & 2 \\
\hline $\begin{array}{l}\text { MHA-TP } \\
\text { Controls }\end{array}$ & & 1 & 3 & 4 & 3 & 3 & 5 & 1 & 2 & 2 \\
\hline RPR & 4 & 1 & 2 & 1 & 2 & 2 & & & & \\
\hline MHA-TP & 0 & 1 & 1 & 1 & 2 & 2 & & & & \\
\hline
\end{tabular}




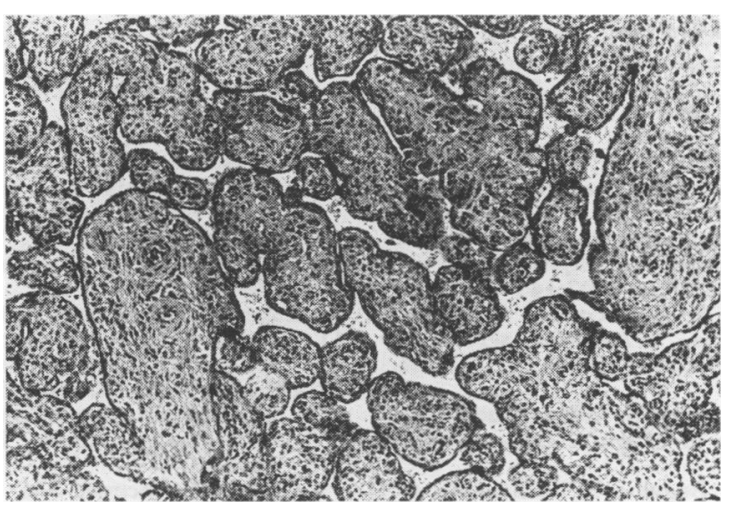

Figure 1 A villus with proliferative and obliterative changes of the vessels. Haematoxylin-eosin $\times 100$.

MHA-TP seroreactivity in RPR reactive sera at titres equal to or above $1: 32$ was significantly associated with stillbirth. This finding agrees with observations made by Watts and colleagues who, in a similar study in Zambia, reported a relationship between stillbirths and high-titre RPR seroreactivity. ${ }^{2}$ High reactivity titres in non-treponemal serological assays, usually indicate early active disease. ${ }^{10}$

The majority of the stillborns had coagulated cord blood. Only in $37 \%$ of the stillborns was serum available for analysis. The prevalence of MHA-TP reactive sera among infants was higher in stillborns than in liveborns. The difference was, however, not statistically significant. RPR reverse titres equal to or above 8 were more frequent among stillborns than among liveborns. The dilemma of the interpretation of such seroreactivities among newborns is to exclude the presence of maternal IgG, which can cross the placenta. Assessment of the presence of IgM in infant's serum was done in 4 sera from stillborns by detection of $T$ pallidum specific IgM by solid-phase haemadsortion (IgM-SPHA). ${ }^{1011}$ Three of four sera were positive (all were found RPR reactive) proving that infection had been acquired in utero.

Our findings do not agree with those of Greenwood et al $^{12}$ in a follow-up study on treponemal infections and the outcome of pregnancy in rural areas of the Gambia. They found high titres of treponemal antibodies in only 13 per thousand stillbirths as compared with 37.5 per thousand in non-seroreactive women. Their findings might be explained by

Figure 2 Placenta from term pregnancy but large immature chorionvilli. Haematoxylin-eosin $\times 25$.

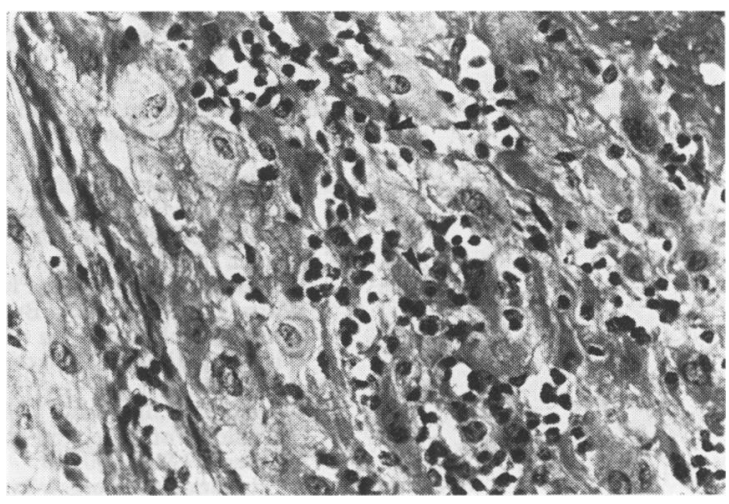

Figure 3 Basal plate with plasma cells. Most of the small dark cells are plasma cells. Haematoxylin-eosin $\times 100$.

the fact that endemic syphilis was prevalent in some regions of the Gambia, including one of the ethnic groups with highest seroreactivity levels.

Yaws was once prevalent in Mozambique, but was eradicated by WHO campaigns in the 1950s. Until now, only occasional cases in one of the northern provinces have been reported. Hence, the MHA-TP seroreactivities found in the present study are likely to reflect current or past syphilis. Screening for syphilis is mandatory at any level of ANC in Maputo, as is treatment, counselling and follow up of seroreactive women. The finding of a high prevalence of seroreactivity to both $R P R$ and MHA-TP is evidence of active syphilis during pregnancy and, as well, of failure of ANC clinics to detect and treat those cases. This emphasises the need to supervise and evaluate the degree of accomplishment of existing management guidelines at ANC clinics.

Only two placentas from MHA-TP seroreactive cases were heavier than normal. Histological acute chorioamnionitis is significantly more frequent among cases, probably due to an acute infection associated with syphilis. Histological changes indicating syphilitic infection, as reported by Qureshi et al and Benirschke and Kaufmann, ${ }^{1314}$ were seen in $32 \%$ of the placentas from seroreactive cases and controls. To confirm the syphilitic origin of these changes demonstration of the presence of spirochetes ${ }^{101314}$ is required. In our study, no spirochetes were visualised in silverstained placental sections. However, spirochetes are difficult to find in silver-stained tissue sections, and this method requires exhaustive searching of many microscopic fields. ${ }^{1014}$ Recently, more specific immunoperoxidase and immunofluorescence techniques have been used on paraffin fixed tissue sections, to identify spirochaetes. ${ }^{1516}$

In conclusion, a high seroprevalence indicative of present or recently past syphilis was found. MHA-TP seroreactivity in RPR reactive sera at reverse titres equal to or above 32 was found to be significantly associated with stillbirth. Morphological changes indicating syphilis infection were found in $32 \%$ placentas histologically studied. Hence syphilis is a risk factor for foetal death in Maputo, Mozambique. 
This study was supported by SAREC (Swedish Agency for Research Co-operation with developing Countries). The authors thank Mrs Zuhura Quiboane, Microbiology Laboratory Technician and Mr Matos Alberto, Pathology Laboratory Technician and Mr Mat

1 Schulz KF, Schulte JM, Berman SM. Maternal health and child survival: opportunities to protect both women an children from the adverse consequences of reproductive tract infections. In: A Germain, ed. Reproductive Tract Infections. New York: Plenum Press, 1992:145-82.

2 Watts E, Larsen SA, Brown S. Case control study at a teaching hospital in Zambia, 1979 1980: serologica investigations for selected infectious agents. WHO 1984, 62(5):803-8.

3 Axemo P, Liliestrand J, Bergström S, Gebre-Medhin M. Etiology of late fetal death in Maputo. Gynecol Obstet Invest 1995;39:103-9.

4 Lindstrand A, Bergström S, Bugalho A, Zanconato G Helgesson AM, Hederstedt B. Prevalence of syphilis infection in Mozambican women with second trimester infection in Mozambican women with second trimester miscarriage and women attending antenatal

5 Liljestrand J, Bergström S, Nieuwenhuis F, Hederstedt B Syphilis during pregnancy in Mozambique. Genitourin Med 1985;61:355-8.

6 Cossa HA, Gloyd S, Vaz RG, Folgosa E, Simbine E, Diniz $M$. Syphilis and HIV infection among displaced pregnan women in rural Mozambique. Intern $\mathcal{F}$ STD AIDS $1994 ; 5: 117-23$
7 Vuylstecke B, Bastos R, Barreto J, Crocitti T, Folgosa E, Mondlane J. High prevalence of sexually transmitted diseases in a rural area in Mozambique. Genitourin Med 1993;69:427-30.

8 EPI INFO. IBM-compatible microcomputers program. Division of surveillance and epidemiology program office CDC, Atlanta, Georgia) \& The global programme on (CDC, Atlanta, Georgia) \& The global pro

9 Malan AF, Woods DL, Van Der Elst CW, Meyer MP. Relative placental weight in congenital syphilis. Placenta 1990;II:3-6.

10 Luger, A. Diagnosis of syphilis. Bull WHO 1981;59:647-54 11 Van Der Sluis JJ. Laboratory techniques in the diagnosis of syphilis: a review. Genitourin Med 1992;68:413-9.

12 Greenwood A, D'Alessandro U, Sisay F, Greenwood BM. Treponemal infection and the outcome of pregnancy in a rural area of Gambia, West Africa. F Inf Dis 1992;166: $842-6$.

13 Qureshi F, Jacques SM, Reys M. Placental histology in syphilis. Human Pathol 1993;24:779-83.

14 Benirschke $\mathrm{K}$, Kauffmman P. Pathology of the Human Placenta. 2nd ed. Springer Verlag, 1990:542-635.

15 Ohyama M, Itani Y, Tanaka Y, Goto A, Sasaki Y. Syphilitic placentitis: demonstration of Treponema pal lidum by immunoperoxidase staining. Archiv $A$ Patho Anat 1990;417:343-5.

16 Ito F, Hunter EF, George RW, Pope V, Larsen SA Specific immunofluorescent staining of pathogenic treponemes with a monoclonal antibody. $f$ Clin Microbio 1992;30:831-8.

\title{
ROYAL MEDICAL BENEVOLENT FUND
}

\section{Help us, help you}

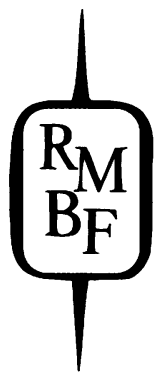

\author{
A nationwide support service \\ for doctors in need, \\ their wives, husbands and children
}

When we take on a case, we never walk away

Donations and enquiries to:

The Secretary, Royal Medical Benevolent Fund

24, King's Road, Wimbledon, London SW19 8QN

Tel: (0181) 5409194 Fax: (0181) 5420494 